\title{
Combinatorial Science and High-Throughput Experiments for Catalysis
}

\author{
Seong Ihl Woo $\cdot$ Soo Ho Kim
}

Published online: 22 December 2009

(C) Springer Science+Business Media, LLC 2009

Inaugural First International Combinatorial Catalysis Symposium (ICCS) was held at KAIST in Daejeon from July 20 to 22,2008 as a post-conference of the 14th International Congress on Catalysis.

Combinatorial technology and high-throughput experimentation (HTE) are becoming a revolutionary research paradigm to discover novel materials more efficiently, rapidly and economically. Conventional one-by-one experiment must be very time-consuming and expensive to perform tremendously large number of experiments which is the case for the development of novel catalysts. During the past decade, automation tools and robotics to prepare catalyst libraries, high-throughput characterization methods and software for informatics and data mining for obtaining the knowledge and optimizing the experimental design have been developed quite successfully. One plenary lecture, two keynote lectures and five invited talks as well as 22 oral and 15 poster presentations reported these recent progresses by world-renowned experts. This symposium provided participants with an open place for exchanging new information and constructive ideas to combinatorial catalysis. Through this symposium our friendship and networking was more intensified for the future.

Among these presentations, 16 papers were selected after extensive reviews and revisions for this issue. The recent advances in the optimum library work-up, experimental design, data mining and high-throughput experiments for catalysis research was reported in this issue. We sincerely hope that these papers will be an important reference for combinatorial catalysis research.
S. I. Woo $(\bowtie) \cdot$ S. H. Kim

Department of Chemical \& Biomolecular Engineering, KAIST,

Daejeon, Korea

e-mail: siwoo@kaist.ac.kr

S. H. Kim

e-mail: soohokim@kaist.ac.kr 